\title{
Inverse Design of Tapers by Bio-Inspired Algorithms
}

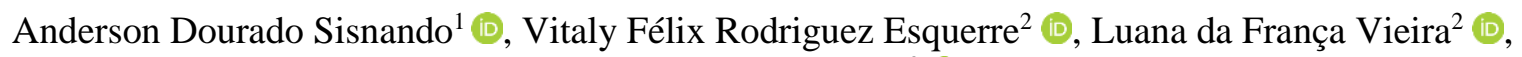 \\ C. E. Rubio-Mercedes ${ }^{3}$ \\ ${ }^{1}$ Federal University of Recôncavo of Bahia, UFRB/CETENS, Bahia, Brazil \\ ${ }^{2}$ Federal University of Bahia, UFBA, Bahia, Brazil \\ ${ }^{3}$ State University of Mato Grosso do Sul, UEMS, Mato Grosso do Sul, Brazil \\ anderson.dourado@ufrb.edu.br,vitaly.esquerre@ufba.br, luanafvieira@yahoo.com.br,cosme@uems.br
}

\begin{abstract}
The efficiency of optical power transferred between two bidimensional waveguides, continuous waveguide and periodic subwavelength waveguide has been designed and optimized by using a taper composed by variable length segments $(0.03$ $0.27 \mu \mathrm{m})$. Waveguides with variable width $(270-330 \mathrm{~nm})$ have been considered and the number of segments on the taper region has been varied from 10 to 15 . The optimized taper was efficiently designed using bio-inspired algorithms based on the genetic algorithms and the artificial immune system. The power coupled has been computed using the frequency domain finite element method. The best solution is $20 \%$ shorter than previously existent tapers with coupling light efficiency above $90 \%$ over a broadband interval of frequencies and it exhibits high fabrication error tolerances.
\end{abstract}

Index Terms - Integrated optics devices; Numerical approximation and analysis; Waveguides; Subwavelength structures.

\section{INTRODUCTION}

The taper waveguide coupler is a passive device composed by a photonic waveguide whose shape or transversal cross section varying along the direction of propagation [1]. Waveguide couplers are generally used to improve or enhance the light coupling efficiency between two waveguides with different transversal sections propagation characteristics. For tapers with long length, the light can be coupled with negligible power loss into the output waveguide [2]-[4]. A drastic reduction of the taper's size will increase the power loss [1], [3].

A good alternative to increase the coupling efficiency between continuous waveguides and periodically segmented waveguides (PSW) is to use a segmented waveguide as a taper waveguide coupler by varying its geometric parameters (length size). One of the main advantages of working with PSW's is the possibility of manipulating the core effective refractive index along the optical circuit. Another desired property is the confinement of light in the PSW filters higher orders modes [5], [6]. In [4]-[8], it is proposed the use of periodically segmented waveguides to change the effective 
guide index and therefore manipulation the field distribution within waveguides.

The aim of this work is to extend the analysis presented in [1], where the number of segments and the segment width were considered constant and the size of taper region was fixed to $4.5 \mu \mathrm{m}$, which is smallest than other previously published. Here, the number of segments in the taper region will be swept from 10 to 15 making the structures of this work ranging from 3.0 to $4.5 \mu \mathrm{m}$ being smaller than those found in [1]. Another contribution of this work is the analysis of the coupling efficiency varying the segments width in the values of $270 \mathrm{~nm}, 300 \mathrm{~nm}$ and $330 \mathrm{~nm}$. The influence of these parameters is analyzed in details aiming to obtain a robust optimized taper with a good tolerance of fabrication errors and a footprint smaller than previously designed tapers.

For the numerical modeling of these tapers, the FEM-2D formulation [9]-[12], is used to compute the field distribution and hence the coupled power can be obtained. On the other hand, the genetic algorithm (GA) and the artificial immune system (AIS) were used for the optimization of several photonic devices such as photonic crystals [11], [12], tapers in conventional waveguides [13], [14], and segmented tapers [1].

Some advantages of the models obtained with AIS and GA are the reduced time and computational effort, its simplicity and also its use in design and synthesis problems [1]. The GA and AIS parameters such as: Individual numbers, number of generations, mutation rates, and affinity have been adopted to be the optimized ones obtained in [1].

The structure to be optimized is displayed in Fig. 1, where $\Lambda, \mathrm{w}$ and a are the period, the width and the length of segments, respectively, $\mathrm{n}_{1}$ is the refractive index of the segments. The PSW is composed by silicon and silica with alternating refractive indices $n_{1}=3.476$ and $n_{2}=1.444$, respectively, and variable width values of $\mathrm{w}=270,300$ and $330 \mathrm{~nm}$. The segments have a fixed period of $\Lambda=300 \mathrm{~nm}$. These parameters are used as the input variables of the bio-inspired algorithms in association with the FEM-2D for computing the fields and power coupling of the optimized structures. Due to the symmetrical feature of the structure along the y direction, only half the geometry was discretized to reduce the computational effort and processing time. Maximizing the coupling efficiency (or transmission coefficient) of PSW optimized taper (varying its length segments), between a straight continuous waveguide (CWG) and a straight PSW, is our main problem [1]. The taper region consists of a variable number of segments between 10 and 15 segments to obtain a small taper. Previously published tapers considering 33 to 200 segments [4] were used for comparison.

The individuals are composed by an array containing the silicon segments lengths $\left\{a_{1}, a_{2}, \ldots, a_{n}\right\}$, where $n=10, \ldots, 15$. An 8 bits coding scheme has been used for each segment length with values in the interval $(30 \mathrm{~nm}, 270 \mathrm{~nm})$ with steps of $0.9375 \mathrm{~nm}$. The end of the taper is at $\mathrm{z}_{1}=0.3 \mathrm{n} \mu \mathrm{m}$.

The manuscript is organized as follows: In Sec. II, we describe the adopted evolutionary algorithms. In Sec. III, we show the respective results founded. Finally, we presented the conclusions in Sec. IV. 


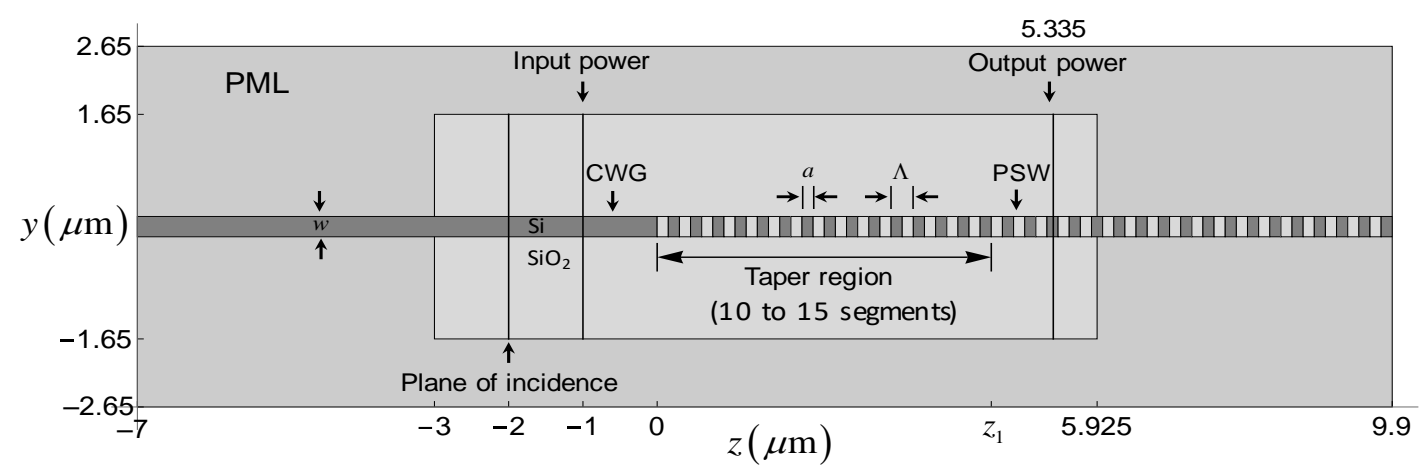

Fig. 1. The structure used for the taper composed by n segments.

To demonstrate the validity and usefulness of artificial immune system and the genetic algorithm for this kind of problem, the evolution and the shape of the best tapers are presented.

\section{BIO-INSPIRED ALGORITHMS}

The bio-inspired algorithms based on the AIS and the GA, reported in [1], are used here and they are shown in Fig. 2.

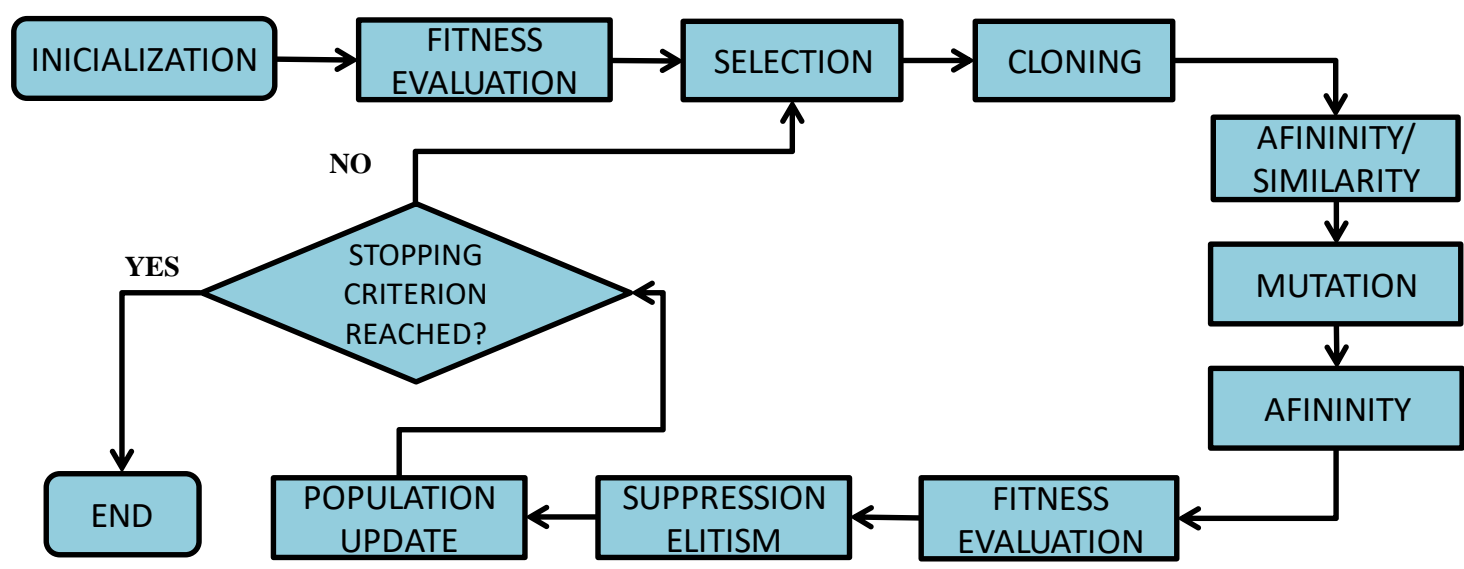

(a)

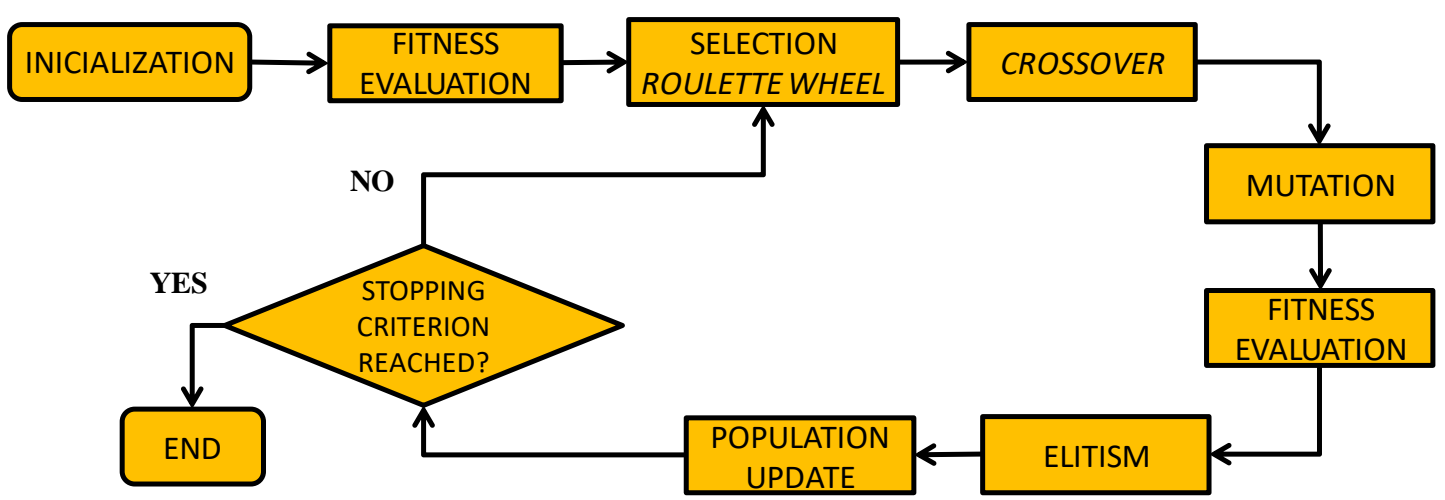

(b)

Fig. 2. The sequential diagrams of the adopted Bio-Inspired algorithms. (a) Based on Artificial Immune System, (b) Based on Genetic Algorithm. 
The steps required for the development and setup of the AIS and GA to treat the taper optimization, in particular, the maximization of power coupling, was adapted from [1]. The proposed algorithms were implemented in MATLAB.

The used bio-inspired algorithms techniques have several steps detailed in [1], with the same Fitness Evaluation for both algorithms (GA and AIS). The fitness of each individual is evaluated by the objective function given by:

$$
\text { Objective Function }=\frac{P_{\text {out }}}{P_{\text {in }}}
$$

where, Pout is the output power coupled to the mode of the output waveguide and Pin is the input power of the mode at the input waveguide.

\section{NUMERICAL RESULTS AND DISCUSSION}

The simulation data used in the bio-inspired algorithms, for each variable, width and number of segments of the segmented taper are: operating wavelength $\lambda=1.55 \mu \mathrm{m}$; segment lengths $a_{i}[0.03 \mu \mathrm{m}$, $0.27 \mu \mathrm{m}], \mathrm{i}=1,2,3 \ldots, \mathrm{n}$; segment refractive index and waveguide $\mathrm{n}_{1}=3.476$; cladding refractive index $\mathrm{n}_{2}=1.444$; and the stop criterion adopted is 200 generations. The computational domain is limited by $\mathrm{z}$ $[-7 \mu \mathrm{m}, 9.9 \mu \mathrm{m}]$ and $\mathrm{y}[0 \mu \mathrm{m},-2.65 \mu \mathrm{m}]$. The initial population size is composed by 10 antibodies and 20 individual for the AIS and GA, respectively. The mutation rate of $3 \%$ has been used for the AIS.

The fundamental Ex polarized mode of the CWG is considered as the incident field of the input taper. We considered three different widths for the CWG, 270nm, 300nm and 330nm. The resulting FEM meshes have about 55,000 elements and 110,000 nodes.

The fitness evolution of the best individuals by using AIS and GA, varying the number of segments (10 to 15$)$ and several width $\mathrm{w}(270,300$ and $330 \mathrm{~nm})$ as a function of the number of generations is shown in Fig. 3. In all cases, the algorithm based on artificial immune system and genetic algorithms presented good convergence.

It is observed that high values of coupling efficiency are obtained with the AIS and GA with a small number of segments $\mathrm{n}$, almost independent of the width $\mathrm{w}$. Table I shows the best coupling efficiency found for each taper configuration with different quantity of segments (10 to 15) as a function of the width variation $(270,300$ and $330 \mathrm{~nm})$. The configuration with width $\mathrm{w}=270 \mathrm{~nm}$ presents the highest coupling efficiency. 


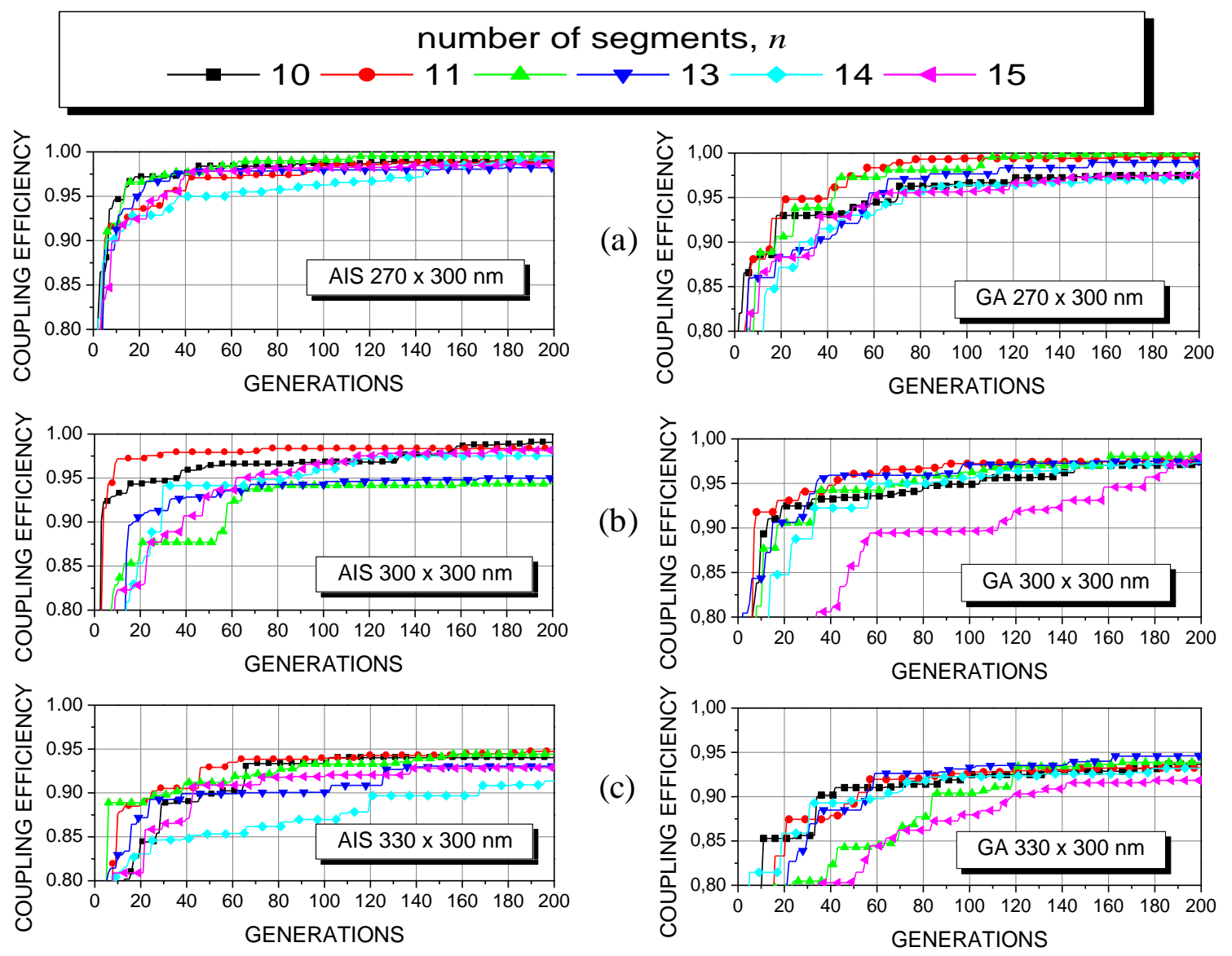

Fig. 3. The evolution of the coupling efficiency of the best individuals for the AIS and for the GA varying the number of segments n (10 to 15), with width w (a) $270 \mathrm{~nm}$, (b) $300 \mathrm{~nm}$ and (c) $330 \mathrm{~nm}$, respectively.

TAble I. Coupling EfFiciency (CE) as a Function of Number of SEgments (NS), FOR AIS AND GA AT $\Lambda=1.55 \mu \mathrm{M}$

\begin{tabular}{cccccccccccccc}
\hline & \multicolumn{1}{c}{$\mathbf{n = 1 0}$} & \multicolumn{2}{c}{$\mathbf{n = 1 1}$} & \multicolumn{2}{c}{$\mathbf{n = 1 2}$} & \multicolumn{2}{c}{$\mathbf{n = 1 3}$} & \multicolumn{2}{c}{$\mathbf{n = 1 4}$} & \multicolumn{2}{c}{$\mathbf{n = 1 5}$} \\
\hline & AIS & GA & AIS & GA & AIS & GA & AIS & GA & AIS & GA & AIS & GA \\
$\mathbf{2 7 0} \times \mathbf{3 0 0} \mathbf{~ n m}$ & 98.96 & 97.48 & 99.10 & 99.60 & $\mathbf{9 9 . 5 3}$ & $\mathbf{9 9 . 9 4}$ & 98.22 & 98.95 & 99.26 & 98.28 & 98.61 & 97.50 \\
$\mathbf{3 0 0} \times \mathbf{3 0 0} \mathbf{~ n m}$ & $\mathbf{9 9 . 0 6}$ & 97.12 & 98.43 & 97.64 & 94.34 & $\mathbf{9 8 . 0 6}$ & 95.00 & 97.53 & 97.53 & 97.30 & 98.16 & 97.98 \\
$\mathbf{3 3 0} \times \mathbf{3 0 0} \mathbf{~ n m}$ & 94.03 & 93.67 & 94.73 & 93.27 & $\mathbf{9 4 . 8 2}$ & 93.87 & 93.06 & $\mathbf{9 4 . 6 2}$ & 91.36 & 93.21 & 92.88 & 91.84 \\
\hline
\end{tabular}

The best optimized tapers obtained by AIS with their respective number of segments (10 to 15) and width w (270, 300 and $330 \mathrm{~nm})$, are displayed in Fig. 4, where we also show the spatial distributions of the field. In Fig. 4(a), it can be observed that high power efficiency is coupled into the taper when the waveguide width is $270 \mathrm{~nm}$. 

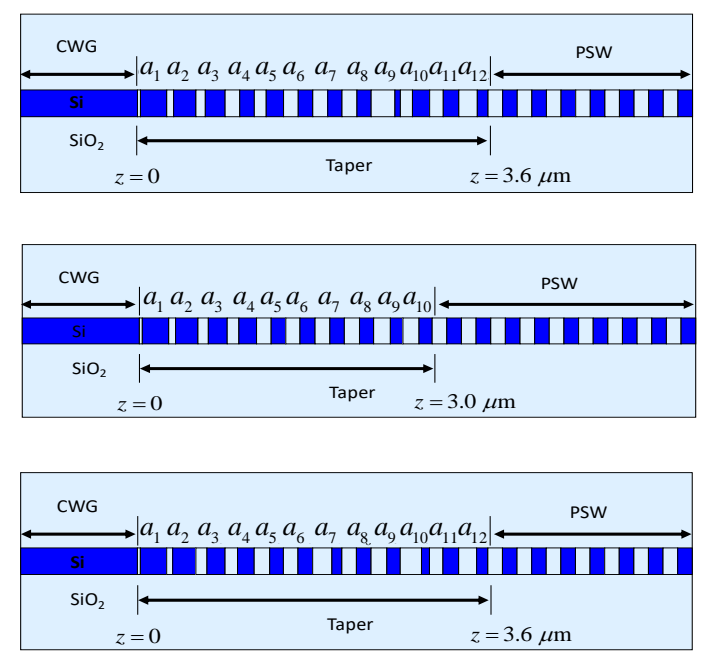
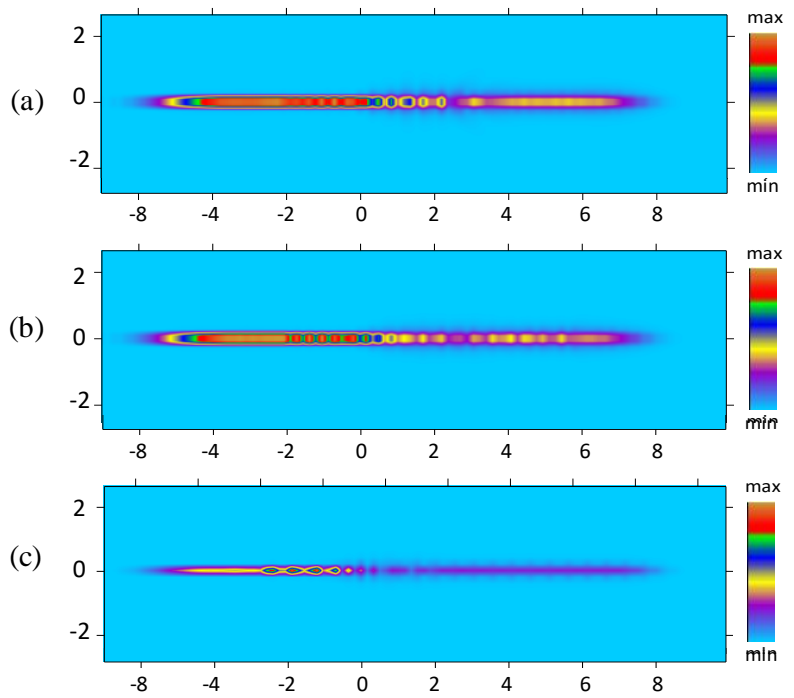

Fig. 4. The optimized PSW taper's shape and Spatial distribution of the field intensity for the best tapers configurations, found by AIS (a) $n=12$ and $w=270 \mathrm{~nm}$, (b) $n=10$ and $w=300 \mathrm{~nm}$ and (c) $n=12$ and $w=330 \mathrm{~nm}$, respectively.

The GA configuration with width $w=270 \mathrm{~nm}$ presents a high coupling efficiency. In Fig. 5, we present the optimized tapers and their respective spatial distributions of the field. It can be observed from Fig. 5(a) that more power is being coupled into the taper with width of $270 \mathrm{~nm}$ than into the others for both algorithms.
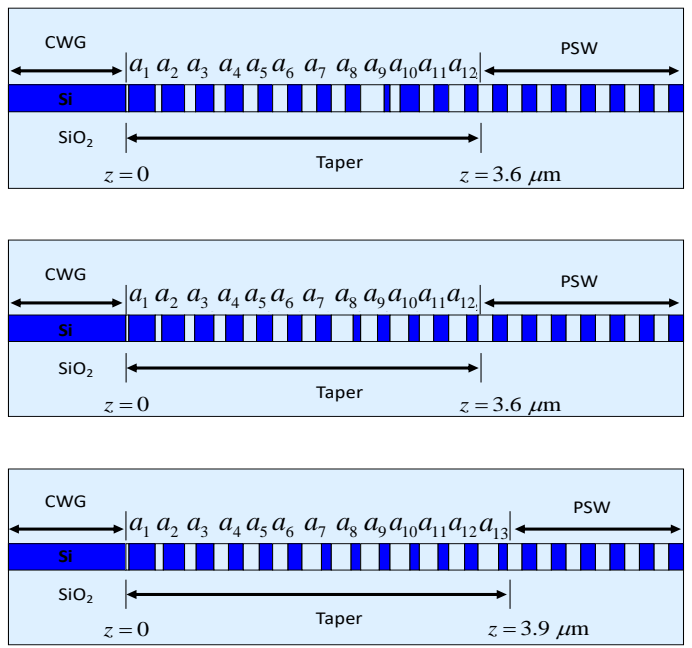

(a)
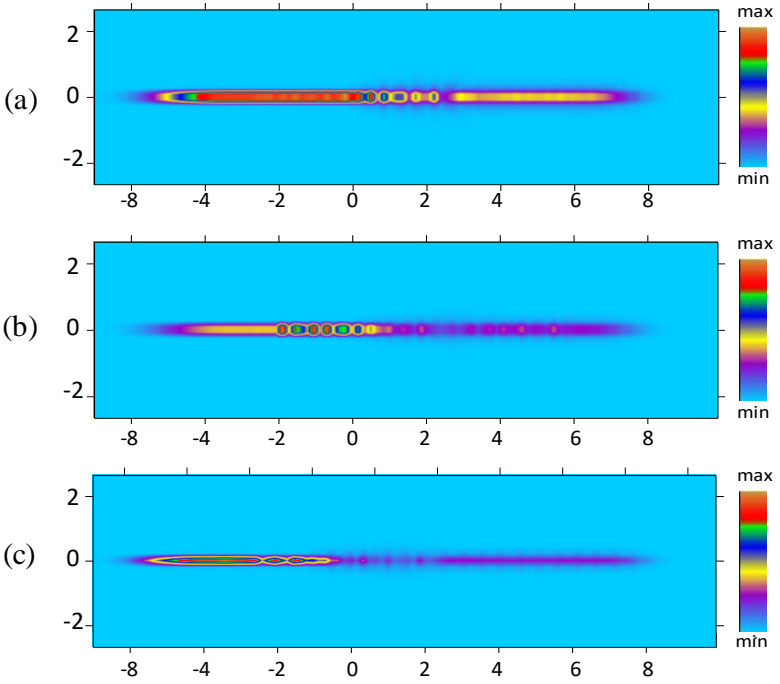

Fig. 5. The optimized PSW taper's shape and Spatial distribution of the field intensity for the best tapers configurations, found by GA (a) n=12 and w=270 nm, (b) n=12 and w=300 nm and (c) n=13 and w=330 nm, respectively.

The segment lengths as a function of the position for the six values marked in bold in Table I are shown in Fig. 6. Note a pattern in the optimized structures: the closest segments located at the input waveguide and ones placed at the output waveguide have a larger filling fraction of silicon and the segments closer to the center of the taper have more silica. The resulting geometries are far different from trivial solutions such as linear or smooth length variation along the taper. 


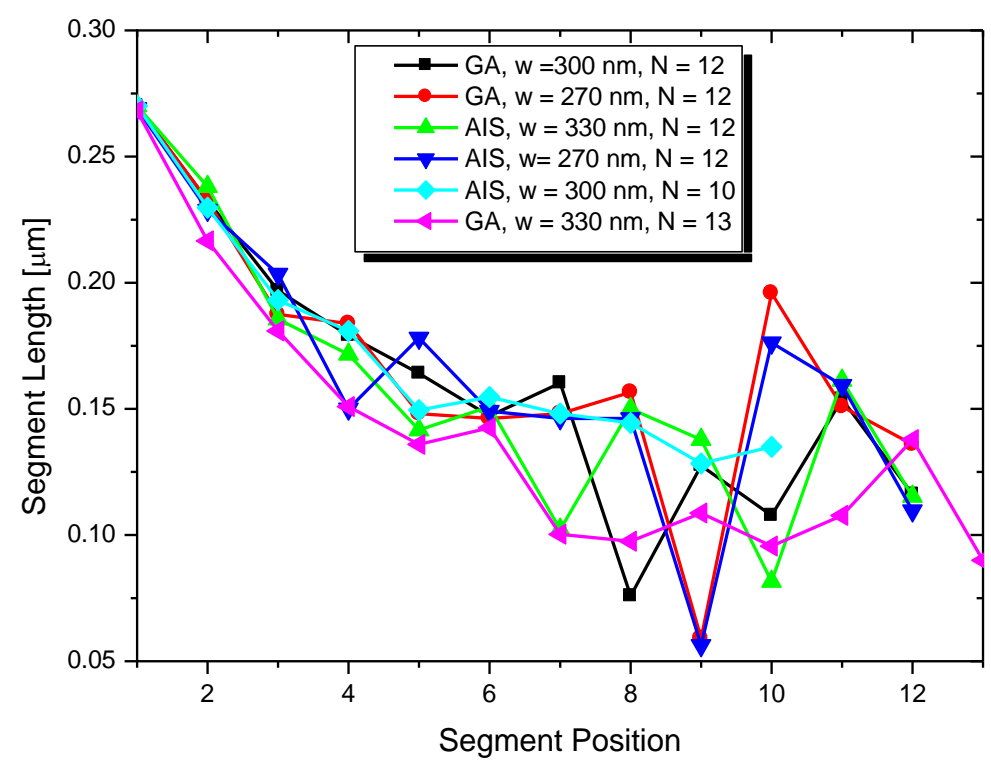

Fig. 6. Segment length vs segment position for the best tapers configurations in Table I.

After optimizing the segmented tapers, using both algorithms, GA and AIS, for the fixed operating wavelength $\lambda=1.55 \mu \mathrm{m}$, their coupling efficiencies have been computed for different operating wavelengths $\lambda[1.50 \mu \mathrm{m} ; 1.60 \mu \mathrm{m}]$, in order to verify the behavior of each of the best optimized tapers. In Fig. 7, we presented the curves of the coupling efficiency as a function of the wavelength for the best structures optimized by using AIS and GA varying the number of segments $n$ (10 to 15) and the width $\mathrm{w}(270,300$ and $330 \mathrm{~nm})$. A variation on the coupling efficiency of about $10 \%$ can be observed, but they are still high (above 83\%).

In addition, the robustness of the designed and optimized tapers with $\mathrm{w}=270 \mathrm{~nm}, 300 \mathrm{~nm}$ and $330 \mathrm{~nm}$, is analyzed by varying (increasing and decreasing) the size of all segment lengths, of the best tapers founded, up to $10 \mathrm{~nm}$. This tolerance is about 10 times the step length used for the segments and it is also compatible with the existent fabrication technologies. The results are displayed in Fig. 9. A variation in coupling efficiency of less than $10 \%$ can be observed. A $0.5 \%$ improvement in coupling efficiency is noted when all segments increase $2 \mathrm{~nm}$. The AIS obtained the best results for $\mathrm{w}=270 \mathrm{~nm}$ while the GA obtained the best results for $\mathrm{w}=270 \mathrm{~nm}$ and $\mathrm{w}=330 \mathrm{~nm}$.

A high coupling efficiency can be observed for the tapers designed using AIS and GA for various wavelengths in this study. Previous reported tapers presents lower coupling efficiency of $81.4 \%$ in [4] while tapers found in [1] and in this work using the AIS and GA presents coupling efficiency above $94 \%$.

The coupler waveguides obtained by the bio-inspired algorithms can be used to design low loss and efficient tapers. 


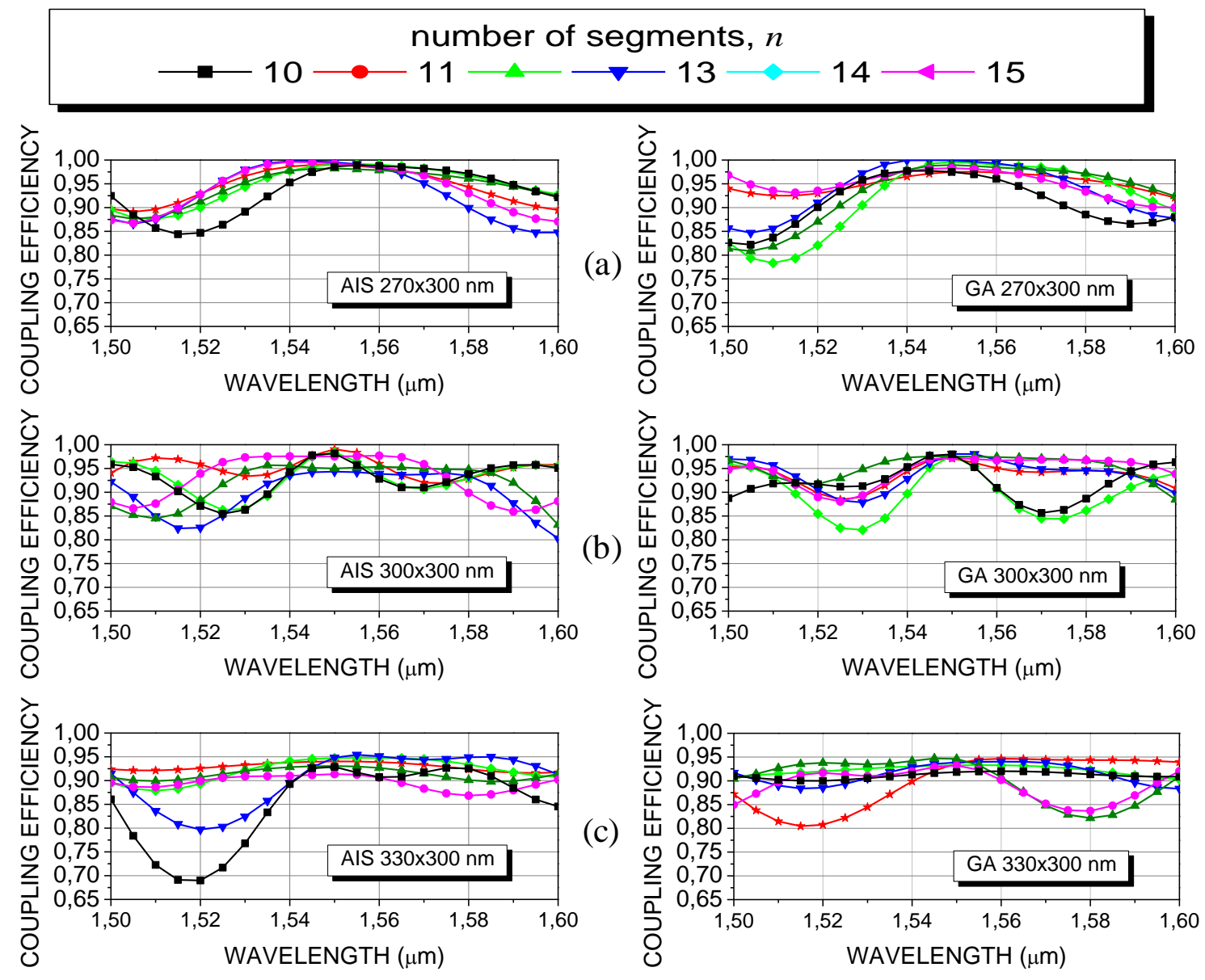

Fig. 7. Coupling efficiency as a function of wavelength operation for the optimized structures by (a) AIS and (b) GA, with $\mathrm{w}=270 \mathrm{~nm}, 300 \mathrm{~nm}$, and $330 \mathrm{~nm}$, respectively.

For the optimized tapers, the reflected power coupled to the mode of the input waveguide is lower than $0.1 \%$. Therefore, we can affirm that most of the non transmitted power is radiated in the taper region. Although the thicknesses of the waveguide has not been taken into account during the optimization process, we include this information indirectly by using the effective index theory in our $2 \mathrm{D}$ model. The core refractive index is $\mathrm{n}=2.52$. This value corresponds to the effective refractive index of a silicon waveguide with thicknesses of $260 \mathrm{~nm}$ surrounded by silica. The coupling efficiencies for the best previously obtained solutions are the following: 98.03\% (GA, $270 \mathrm{~nm} x$ $300 \mathrm{~nm}$ ), 98.10\% (AIS, $270 \mathrm{~nm}$ x $300 \mathrm{~nm}$ ), 93.03\% (GA, $300 \mathrm{~nm}$ x $300 \mathrm{~nm}$ ), 96.47\% (AIS, $300 \mathrm{~nm}$ x $300 \mathrm{~nm}$ ), 82.24\% (GA, $330 \mathrm{~nm} \times 300 \mathrm{~nm}$ ), and $85.17 \%$ (AIS, $330 \mathrm{~nm} \times 300 \mathrm{~nm}$ ). It is possible to observe high transmission for thin waveguides and a reduction of the efficiency for wider waveguides. Consequently, the 2D approach can be used to obtain an insight of the behavior of 3D taper.

Moreover, the wavelength dependence of the GA optimized waveguide, under the effective index theory, with width $\mathrm{w}=270 \mathrm{~nm}$ is presented in Fig. 8. It can be seen that the coupling efficiency is above $96 \%$ in all the interval of wavelengths. 


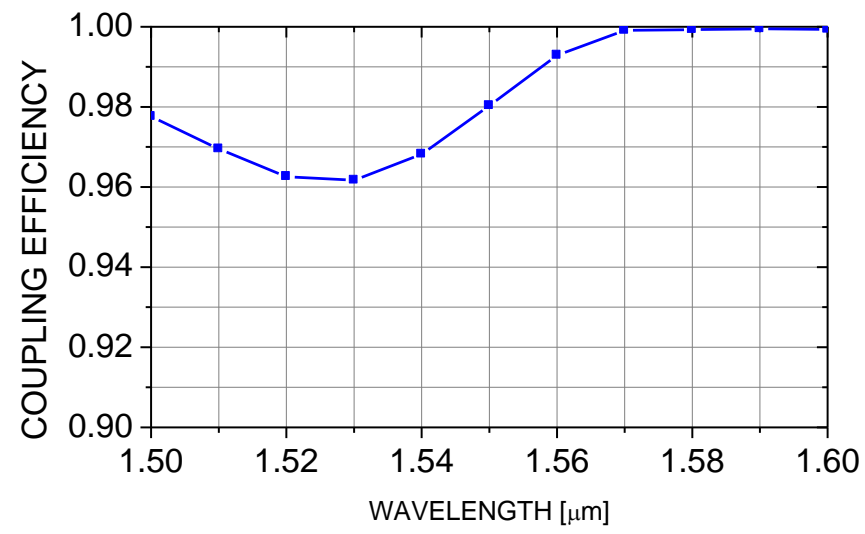

Fig. 8. The coupling efficiency as a function of wavelength operation for the optimized structures by GA, with $\mathrm{w}=270 \mathrm{~nm}$, using the effective index theory for the core refractive index, $\mathrm{n}_{1}=2.52$.

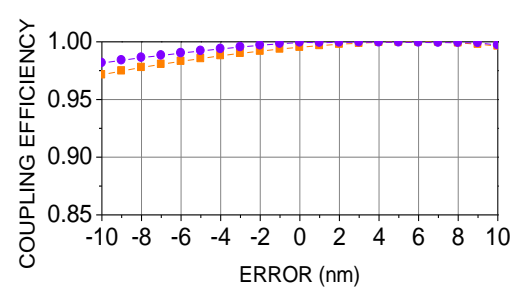

(a)

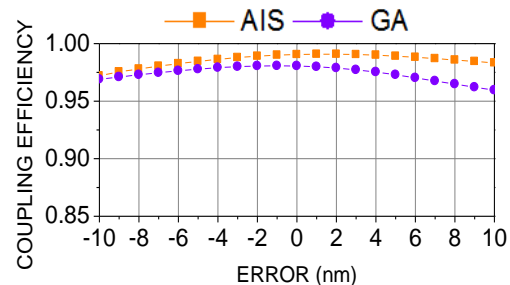

(b)

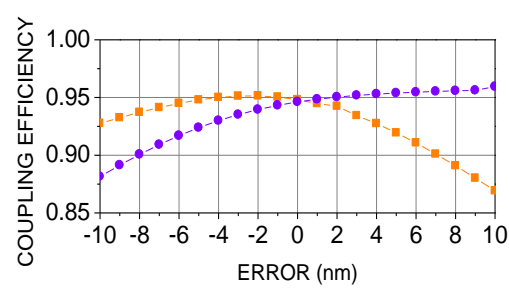

(c)

Fig. 9. Coupling efficiency spectrum with a random variation of the optimized segments length size at $1.55 \mu \mathrm{m}$, (a) $\mathrm{w}=270 \mathrm{~nm},(\mathrm{~b}) \mathrm{w}=300 \mathrm{~nm}$ and (c) $\mathrm{w}=330 \mathrm{~nm}$, respectively.

Finally, we run the experiment multiple times due to the stochastic nature of the proposed methods in order to demonstrate the convergence of the proposed algorithms. We considered the case of 15 segments because it was the longest taper and it could present more stochastic behavior. We run the experiments 10 times. The obtained results are shown in Fig. 10.

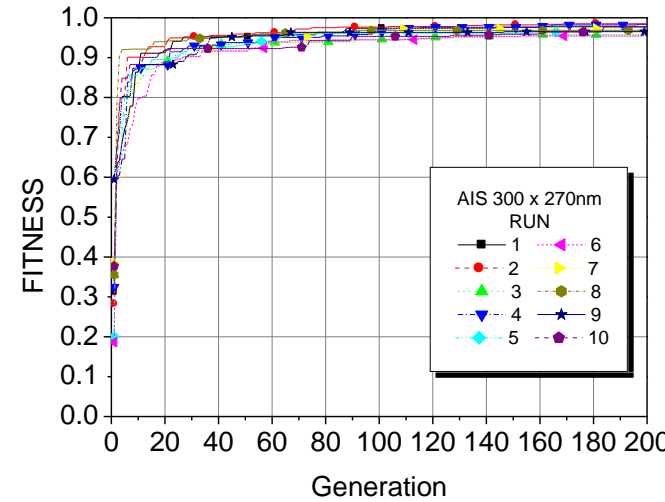

(a)

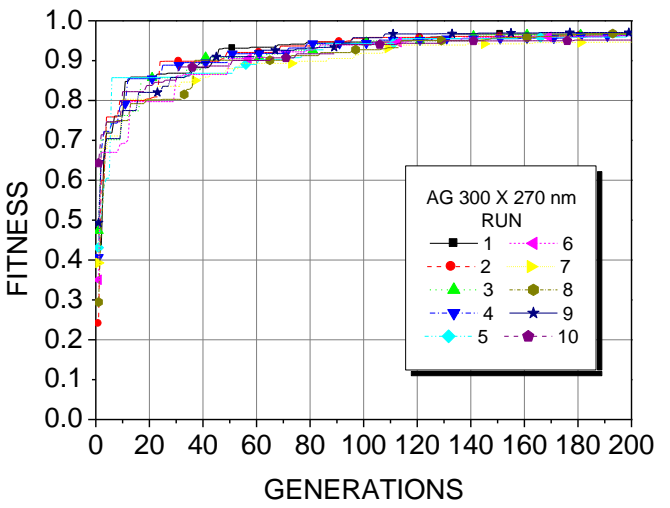

(b)

Fig. 10. Fitness evolution after running the AG and AIS algorithms 10 times.

We can observe that both algorithms converge to values above $96 \%$ after running them ten times 
during 200 generations with a mean efficiency value of $96.25 \%$ with standard deviation of $0.787 \%$ for the AG while for the SIA, the mean value of the efficiency was $97.12 \%$ with standard deviation of $0.999 \%$. It is expected a similar behavior for all the analyzed tapers.

\section{CONCLUSIONS}

In conclusion, the optimization of planar waveguide couplers using PSW composed by silicon and silica, using the 2D-FEM in association with bio-inspired algorithms based on the AIS and the GA is presented. The results presented are better than the ones previously reported in literature considering sizes and materials with refractive indexes close to the ones used in this work.

It can be observed a pattern in the optimized structures: the closest segments of the input waveguide and the output waveguide have a larger filling fraction of silicon and the segments closer to the center of the taper have more silica.

A new set of tapers are presented, varying the number of the segments (10-15) and varying the width $(270,300$ and $330 \mathrm{~nm})$ and the optimized structures with $\mathrm{w}=270 \mathrm{~nm}$ show better results than the others presented in [1] and [4]. The footprint of the best solution is $20 \%$ smaller than previously ones reported in the literature.

Coupling efficiencies above $94 \%$ have been found for specific operating wavelengths and above $84 \%$ for the wavelength interval [1.5 $\mu \mathrm{m}-1.6 \mu \mathrm{m}]$. In addition, the designed models can be used to design optical waveguide couplers considering different materials, number of sections, period variation, different waveguides and 3D simulations. The proposed solutions exhibit high fabrication error tolerances.

\section{ACKNOWLEDGMENT}

The authors acknowledge the financial support of UFBA, UFRB/CETENS, CAPES (001), FAPESB (079/2016) and CNPq Process: 309100/2018-6.

\section{REFERENCES}

[1] A. Dourado-Sisnando, V. F. Rodríguez-Esquerre, C. E. Rubio-Mercedes, L. F. Vieira and I. M. T. Ruffini, "Power Coupling Optimization in 2D Waveguides by Evolutionary Algorithms," IEEE Photon. Technol. Lett, vol. 27, no. 14, pp. 1561-1564, 2015.

[2] T. Felici, R. Black, and D. Gallagher, "Recent advances and results in waveguide shape optimization techniques," in Lasers and Electro-Optics Society, 2002, pp. 831-832.

[3] T. Felici, and H. W. Engl, "On shape optimization of optical waveguides using inverse problem techniques," IOPScience Inverse Problems, vol. 17, pp. 1141, 2001.

[4] P. Cheben, D-X. Xu, S. Janz, and A. Densmore, "Subwavelength waveguide grating for mode conversion and light coupling in integrated optics," Opt. Exp., vol. 14, no. 11, pp. 4695-4702, 2006.

[5] P. J. Bock, P. Cheben, J. H. Schmid, J. Lapointe, A. Delâge, S. Janz, G. C. Aers, D. Xu, A. Densmore, and T. J. Hall, "Subwavelength grating periodic structures in silicon-on-insulator: A new type of microphotonic waveguide," Opt. Exp., vol. 18, no. 19, pp. 20251-20262, 2010.

[6] P. J. Bock, P. Cheben, J. H. Schmid, J. Lapointe, A. Delâge, D. Xu, S. Janz, A. Densmore, and T. J. Hall, "Subwavelength grating crossing for silicon wire waveguides," Opt. Exp., vol. 18, no. 15, pp. 16146-16155, 2010.

[7] D. Nir, Z. Weissman, S. Ruschin, and A. Hardy, "Periodically segmented waveguide in TiLiNbO3," Opt. Lett., vol. 19, no. 21, pp. 1732 - 1734, 1994.

[8] Z. Weissman, and A. Hardy, "2-d mode tapering via tapered channel waveguide segmentation," Electronics Lett., vol. 28, no. 16, pp. 1514-1516, 1992. 
[9] C. E. Rubio-Mercedes, V. F. Rodríguez-Esquerre, I. T. Lima and H. E. Hernández-Figueroa, “Analysis of Straight Periodic Segmented Waveguide Using the 2-D Finite Element Method," J. Lightw. Technol.,vol. 32, no. 11, pp. 21632169, 2014.

[10] C. E. Rubio-Mercedes and H. E. Hernández-Figueroa, "Padé boundary conditions for the finite-element solution of arbitrary planar junctions," J. Lightw. Technol., vol. 22, no. 2, pp. 669-676, 2004.

[11] A. Dourado-Sisnando, L. F. Vieira, V. F. Rodriguez-Esquerre and F. G. S. Silva, "Artificial Immune System Optimisation of Complete Bandgap of Bidimensional Anisotropic Photonic Crystals," IET Optoelectronics, vol. 9, no. 6, pp. 333-340, 2015.

[12] G. N. Malheiros-Silveira, V. F. Rodríguez-Esquerre, and H. E. Hernández-Figueroa, "Strategy of Search and Refinement by GA in 2-D Photonic Crystals with Absolute PBG," IEEE J. Quantum Electron., vol. 47, no. 4, pp. 431437, 2011.

[13] A. Dourado-Sisnando, I. M. T. Ruffini, R. R. S. Andrade, V. F. Rodríguez-Esquerre, and C. E. Rubio-Mercedes, "Power Coupling Optimization by Artificial Immune System," in Integrated Photonics Research, Silicon and Nano Photonics, San Diego, United States of America, 2014, presentation number: IT1A.2.

[14] A. Dourado-Sisnando, L. F. Vieira, V. F. Rodríguez-Esquerre and C. E. Rubio-Mercedes, "Taper Design and Optimization by Evolutionary Algorithms," in Latin America Optics and Photonics Conference, Cancún, México, 2014, presentation number: LM4A.3. 